PROCEEDINGS OF THE

AMERICAN MATHEMATICAL SOCIETY

Volume 128, Number 11, Pages 3405-3409

S 0002-9939(00)05465-4

Article electronically published on May 11, 2000

\title{
REMARKS ON COMMUTING EXPONENTIALS IN BANACH ALGEBRAS, II
}

\author{
CHRISTOPH SCHMOEGER
}

(Communicated by Dale Alspach)

\begin{abstract}
Suppose that $a$ and $b$ are elements of a complex unital Banach algebra such that the spectrum of $a$ is $2 \pi i$-congruence-free and $e^{a} e^{b}=e^{b} e^{a}$. We show that then $a b-b a$ is the sum of nilpotent elements. If $r(b)$ denotes the spectral radius of $b$, then we show that the additional assumption $r(b)<2 \pi$ implies that
\end{abstract}

$$
b(a b-b a)^{2}=(a b-b a)^{2} b .
$$

The present paper is a continuation of our previous paper [4]. Let $\mathcal{A}$ denote a complex unital Banach algebra. By $\mathcal{L}(\mathcal{A})$ we denote the Banach algebra of all bounded linear operators on $\mathcal{A}$. For $x \in \mathcal{A}$ the spectrum and the spectral radius of $x$ are denoted by $\sigma(x)$ and $r(x)$, respectively. The map $\delta_{x}: \mathcal{A} \rightarrow \mathcal{A}$, defined by

$$
\delta_{x}(c)=c x-x c \quad(c \in \mathcal{A}),
$$

is called the inner derivation determined by $x$. From $\left\|\delta_{x}(c)\right\| \leq 2\|c\|\|x\|$ it follows that $\delta_{x} \in \mathcal{L}(\mathcal{A})$.

If $T \in \mathcal{L}(\mathcal{A})$, then $N(T)$ denotes the kernel of $T$.

If $\Omega_{1}$ and $\Omega_{2}$ are subsets of $\mathbb{C}$, then $\Omega_{1}-\Omega_{2}:=\left\{\lambda-\mu: \lambda_{1} \in \Omega_{1}, \lambda_{2} \in \Omega_{2}\right\}$.

Proposition 6.4.8 in [2] shows that

$$
\sigma\left(\delta_{x}\right) \subseteq \sigma(x)-\sigma(x)=\{\lambda-\mu: \lambda, \mu \in \sigma(x)\}
$$

and

$$
e^{\delta_{x}}(c)=e^{-x} c e^{x} \quad \text { for all } c \in \mathcal{A} \text {. }
$$

Define the entire function $f: \mathbb{C} \rightarrow \mathbb{C}$ by

$$
f(\lambda)= \begin{cases}\lambda^{-1}\left(e^{\lambda}-1\right), & \text { if } \lambda \neq 0, \\ 1, & \text { if } \lambda=0 .\end{cases}
$$

Since $\lambda f(\lambda)=f(\lambda) \lambda=e^{\lambda}-1$, we get for $x \in \mathcal{A}$

$$
f\left(\delta_{x}\right) \delta_{x}=e^{\delta_{x}}-I
$$

thus, by (2),

$$
f\left(\delta_{x}\right)\left(\delta_{x}(c)\right)=e^{-x} c e^{x}-c \text { for all } c \in \mathcal{A} .
$$

Received by the editors August 28, 1998 and, in revised form, January 22, 1999.

1991 Mathematics Subject Classification. Primary 46H99.

Key words and phrases. Commuting exponentials. 
A set $\Omega \subseteq \mathbb{C}$ is called $2 \pi i$-congruence-free if $\lambda, \mu \in \Omega$ and $\lambda \equiv \mu(\bmod 2 \pi i)$ imply that $\lambda=\mu$.

The following result is due to E. M. E. Wermuth ([6]). See also [4 for a very short proof using (3).

Theorem 1. Let $a, b \in \mathcal{A}$. Suppose that $\sigma(a)$ and $\sigma(b)$ are $2 \pi i$-congruence-free and that $e^{a} e^{b}=e^{b} e^{a}$. Then $a b=b a$.

In [3, Corollary 1] we have shown that if $A$ and $B$ are bounded self-adjoint operators on a Hilbert space, then

$$
e^{A} e^{B}=e^{A+B} \quad \text { if and only if } A B=B A .
$$

Even in the case of two-dimensional matrices the equivalence (4) does not hold under the weaker assumption of $2 \pi i$-congruence-free spectra ([5]). But we have:

Theorem 2. Let $a, b \in \mathcal{A}$. Suppose that $\sigma(a+b)$ is $2 \pi i$-congruence-free. If

$$
e^{a} e^{b}=e^{a+b}=e^{b} e^{a},
$$

then $a b=b a$.

Proof. Since $\sigma(a+b)$ is $2 \pi i$-congruence-free, (1) shows that $f$ does not vanish on $\sigma\left(\delta_{a+b}\right)$; hence $f\left(\delta_{a+b}\right)$ is invertible in $\mathcal{L}(\mathcal{A})$. From (3) we get

$\delta_{a+b}\left(e^{a}\right)=f\left(\delta_{a+b}\right)^{-1}\left(e^{-(a+b)} e^{a} e^{a+b}-e^{a}\right)=f\left(\delta_{a+b}\right)^{-1}\left(e^{-b} e^{-a} e^{a} e^{b} e^{a}-e^{a}\right)=0 ;$

thus $e^{a}(a+b)=(a+b) e^{a}$. Therefore $b e^{a}=e^{a} b$. This gives

$\delta_{a+b}(b)=f\left(\delta_{a+b}\right)^{-1}\left(e^{-(a+b)} b e^{a+b}-b\right)=f\left(\delta_{a+b}\right)^{-1}\left(e^{-b} e^{-a} b e^{a} e^{b}-b\right)=0$.

Hence $b(a+b)=(a+b) b$. This shows that $b a=a b$.

Corollary 1. If $A$ is a bounded linear operator on a complex Hilbert space, then

$$
e^{A} e^{A^{*}}=e^{A+A^{*}}=e^{A^{*}} e^{A} \quad \text { if and only if } A \text { is normal. }
$$

Proof. Since $A+A^{*}$ is self-adjoint, $\sigma\left(A+A^{*}\right) \subseteq \mathbb{R}$. Hence $\sigma\left(A+A^{*}\right)$ is $2 \pi i$ congruence-free.

For our main results in this paper we need the following propositions.

Proposition 1. Let $c, d, x \in \mathcal{A}$ and $\lambda_{0}, \mu_{0} \in \mathbb{C} \backslash\{0\}$. If $c x-x c=\lambda_{0} c$ and $d x-x d=$ $\mu_{0} d$, then:

(a) $(d c) x-x(d c)=\left(\mu_{0}+\lambda_{0}\right) d c$;

(b) $c^{n} x-x c^{n}=n \lambda_{0} c^{n} \quad$ for all $n \in \mathbb{N}$;

(c) $c^{n}=0$ for all $n \in \mathbb{N}$ with $n>2\|x\|\left|\lambda_{0}\right|^{-1}$.

Proof. (a) From $x c=c x-\lambda_{0} c$ and $x d=d x-\mu_{0} d$ we derive

$$
\begin{aligned}
(d c) x-x(d c) & =(d c) x-(x d) c \\
& =(d c) x-\left(d x-\mu_{0} d\right) c \\
& =(d c) x-d(x c)+\mu_{0} d c \\
& =(d c) x-d\left(c x-\lambda_{0} c\right)+\mu_{0} d c \\
& =(d c) x-(d c) x+\left(\lambda_{0}+\mu_{0}\right) d c=\left(\lambda_{0}+\mu_{0}\right) d c .
\end{aligned}
$$

(b) follows from (a) by induction.

(c) If $n\left|\lambda_{0}\right|>2\|x\|$, then $n\left|\lambda_{0}\right|>\left\|\delta_{x}\right\|$; thus $n \lambda_{0} \notin \sigma\left(\delta_{x}\right)$. Hence, by (b), $c^{n}=0$. 
Remark. Proposition 1 is valid for not necessarily inner derivations $D: \mathcal{A} \rightarrow \mathcal{A}$.

Recall that the entire function $f$ is defined by $f(\lambda)=\lambda^{-1}\left(e^{\lambda}-1\right)$ if $\lambda \neq 0$ and $f(0)=1$. We use the following notation for $x \in \mathcal{A}$ :

$$
M_{x}=\left\{\lambda \in \sigma\left(\delta_{x}\right): f(\lambda)=0\right\} .
$$

Observe that if $M_{x}=\emptyset$, then $f\left(\delta_{x}\right)$ is invertible in $\mathcal{L}(\mathcal{A})$. Observe also that if $\sigma(x)$ is $2 \pi i$-congruence-free, then, by (1), $M_{x}=\emptyset$.

Since $\sigma\left(\delta_{x}\right)$ is compact it follows that $M_{x}$ is a finite set. From the definition of $f$ it is clear that each $\lambda \in M_{x}$ is a simple zero of $f$ and that there is $k \in \mathbb{Z} \backslash\{0\}$ and that $\lambda=2 k \pi i$.

Proposition 2. Let $x \in \mathcal{A}$ and let $n$ denote the smallest integer with $n>\frac{r(x)}{\pi}$. We have:

(a) $M_{x}$ has at most $2 n-2$ elements;

(b) if $M_{x} \neq \emptyset$ and $M_{x}=\left\{\lambda_{1}, \ldots, \lambda_{k}\right\}$ with $k \leq 2 n-2$ and $\lambda_{j} \neq \lambda_{l}$ for $j \neq l$, then

$$
N\left(f\left(\delta_{x}\right)\right)=N\left(\delta_{x}-\lambda_{1} I\right) \oplus \cdots \oplus N\left(\delta_{x}-\lambda_{k} I\right)
$$

and for each $z \in N\left(f\left(\delta_{x}\right)\right)$ there are $u_{1}, \ldots, u_{k} \in \mathcal{A}$ such that

$$
z=u_{1}+\cdots+u_{k}, \quad u_{j} \in N\left(\delta_{x}-\lambda_{j} I\right) \text { and } u_{j}^{n}=0
$$

for $j=1, \ldots, k$.

Proof. (a) Suppose that $M_{x} \neq \emptyset$. If $\lambda \in M_{x}$, then $\lambda=2 k \pi i$ for some $k \in \mathbb{Z} \backslash\{0\}$. By (11) there are $\alpha, \beta \in \sigma(x)$ with $\lambda=\alpha-\beta$. Thus

$$
2|k| \pi=|\lambda|=|\alpha-\beta| \leq|\alpha|+|\beta| \leq 2 r(x)<2 n \pi ;
$$

hence $|k| \leq n-1$. Therefore

$$
M_{x} \subseteq\{ \pm 2 \pi i, \pm 4 \pi i, \ldots, \pm 2(n-1) \pi i\} .
$$

(b) Recall that each $\lambda_{j}$ is a simple zero of $f$; hence there is an entire function $g$ such that

$$
f(\lambda)=g(\lambda)\left(\lambda-\lambda_{1}\right) \cdots\left(\lambda-\lambda_{k}\right) .
$$

It follows that $g\left(\delta_{x}\right)$ is invertible in $\mathcal{L}(\mathcal{A})$ and

$$
f\left(\delta_{x}\right)=g\left(\delta_{x}\right)\left(\delta_{x}-\lambda_{1} I\right) \cdots\left(\delta_{x}-\lambda_{k} I\right) .
$$

Satz 80.3 in [1] gives now

$$
N\left(f\left(\delta_{x}\right)\right)=N\left(\delta_{x}-\lambda_{1} I\right) \oplus \cdots \oplus N\left(\delta_{x}-\lambda_{k} I\right) .
$$

Take $z \in N\left(f\left(\delta_{x}\right)\right)$. Then there are $u_{j} \in N\left(\delta_{x}-\lambda_{j} I\right) \quad(j=1, \ldots, k)$ with $z=u_{1}+\cdots+u_{k}$. Let $j \in\{1, \ldots, k\}$. Proposition 1 (b) shows that

$$
u_{j}^{n} x-x u_{j}^{n}=n \lambda_{j} u_{j}^{n} .
$$

Suppose that $u_{j}^{n} \neq 0$. Then $n \lambda_{j} \in \sigma\left(\delta_{x}\right)$. As in the proof of (a) we derive

$$
n\left|\lambda_{j}\right| \leq n 2 r(x)<2 n \pi
$$

hence $\left|\lambda_{j}\right|<2 \pi$, a contradiction since $\lambda_{j} \neq 0$.

Let $x \in \mathcal{A}$ and $r(x)<\pi$. Then $\sigma(x)$ is $2 \pi i$-congruence-free and hence $N\left(f\left(\delta_{x}\right)\right)=$ $\{0\}$. The following theorem deals with the case where $\pi \leq r(x)<2 \pi$. 
Theorem 3. Let $x \in \mathcal{A}$ and suppose that $\pi \leq r(x)<2 \pi$. Then

$$
x z^{2}=z^{2} x \quad \text { for each } z \in N\left(f\left(\delta_{x}\right)\right) .
$$

There are the following three possibilities:

(a) $M_{x}=\emptyset$. In this case we have $N\left(f\left(\delta_{x}\right)\right)=\{0\}$.

(b) $M_{x}=\{\lambda\}$ with $\lambda=2 \pi i$ or $\lambda=-2 \pi i$. In this case we have $z^{2}=0$ for each $z \in N\left(f\left(\delta_{x}\right)\right)$.

(c) $M_{x}=\left\{\lambda_{1}, \lambda_{2}\right\}$ with $\lambda_{1}=2 \pi i=-\lambda_{2}$. In this case (5) holds.

Proof. From Proposition 2 we know that $M_{x}$ has at most two elements. Suppose that $M_{x} \neq \emptyset$. Take $\lambda \in M_{x}$; hence $\lambda=2 k \pi i \quad(k \in \mathbb{Z} \backslash\{0\})$. As in the proof of Proposition 2 (a) we see that $|k| \leq 1$; thus $\lambda= \pm 2 \pi i$.

Case 1: $\quad M=\left\{\lambda_{1}\right\}$. Proposition 2 (b) gives

$$
N\left(f\left(\delta_{x}\right)\right)=N\left(\delta_{x}-\lambda_{1} I\right) \text { and } z^{2}=0 \text { for each } z \in N\left(f\left(\delta_{x}\right)\right) .
$$

Case 2: $\quad M=\left\{\lambda_{1}, \lambda_{2}\right\}$. Then $\lambda_{1}=2 \pi i=-\lambda_{2}$. Let $z \in N\left(f\left(\delta_{x}\right)\right)$. By Proposition 2 (b) there are $u_{j} \in N\left(\delta_{x}-\lambda_{j} I\right)$ with $u_{j}^{2}=0 \quad(j=1,2)$ and $z=u_{1}+u_{2}$.

Proposition 1 (a) gives

$$
u_{1} u_{2} x-x u_{1} u_{2}=\left(\lambda_{1}+\lambda_{2}\right) u_{1} u_{2}=0 . u_{1} u_{2}=0
$$

thus $x u_{1} u_{2}=u_{1} u_{2} x$. A similar argument shows that $x u_{2} u_{1}=u_{2} u_{1} x$. From $z^{2}=u_{1}^{2}+u_{1} u_{2}+u_{2} u_{1}+u_{2}^{2}=u_{1} u_{2}+u_{2} u_{1}$ it follows then that

$$
x z^{2}=x u_{1} u_{2}+x u_{2} u_{1}=u_{1} u_{2} x+u_{2} u_{1} x=z^{2} x .
$$

Theorem 4. Let $a, b \in \mathcal{A}$ and $e^{a} e^{b}=e^{b} e^{a}$. Suppose that $\sigma(a)$ is $2 \pi i$-congruencefree. Then $a b-b a$ is the sum of nilpotent elements. If in addition $r(b)<2 \pi$, then

$$
b(a b-b a)^{2}=(a b-b a)^{2} b .
$$

Proof. Since $f\left(\delta_{a}\right)$ is invertible in $\mathcal{L}(\mathcal{A})$ we get from

$$
f\left(\delta_{a}\right)\left(\delta_{a}\left(e^{b}\right)\right)=e^{-a} e^{b} e^{a}-e^{b}=0
$$

that $a e^{b}=e^{b} a$. Therefore

$$
f\left(\delta_{b}\right)\left(\delta_{b}(a)\right)=e^{-b} a e^{b}-a=0 ;
$$

hence $a b-b a=\delta_{b}(a) \in N\left(f\left(\delta_{b}\right)\right)$. Use Proposition 2 Theorem 1 and Theorem 3 to complete the proof.

With the aid of Theorem 3 we can prove a further result concerning exponentials in Banach algebras.

Theorem 5. Suppose that $a, b \in \mathcal{A}$ and $e^{a}=e^{b}$.

(a) If $\sigma(a)$ is $2 \pi i$-congruence-free, then $a b=b a$.

(b) If $\pi \leq r(a)<2 \pi$, then $a(a b-b a)^{2}=(a b-b a)^{2} a$.

Proof. From (3) we get

$$
f\left(\delta_{a}\right)(b a-a b)=f\left(\delta_{a}\right)\left(\delta_{a}(b)\right)=e^{-a} b e^{a}-b=e^{-b} b e^{b}-b=0 .
$$

Hence $a b-b a \in N\left(f\left(\delta_{a}\right)\right)$.

(a) If $\sigma(a)$ is $2 \pi i$-congruence-free we have $N\left(f\left(\delta_{a}\right)\right)=\{0\}$; thus $a b=b a$.

(b) follows from Theorem 3 
For our next result we denote by 1 the unit of $\mathcal{A}$.

Theorem 6. Let $a, b \in \mathcal{A}$, let $e^{a}=e^{b}$ and let $\sigma(a)$ and $\sigma(a)-\sigma(b)$ be $2 \pi i$ congruence-free. Then there is some $k \in \mathbb{Z}$ with

$$
a-b=(2 k \pi i) \mathbf{1} \text {. }
$$

Proof. From Theorem 5 (a) we get $a b=b a$. Thus $e^{a-b}=1$. Put $c=a-b$ and define $g: \mathbb{C} \rightarrow \mathbb{C}$ by $g(\lambda)=e^{\lambda}-1$. Then $g(c)=0$. Take $\lambda_{1}, \lambda_{2} \in \sigma(c)$. Since $a$ and $b$ commute, we get from [3, Theorem 11.23] that $\sigma(c) \subseteq \sigma(a)-\sigma(b)$; thus $\lambda_{1}, \lambda_{2} \in \sigma(a)-\sigma(b)$. The spectral mapping theorem gives $e^{\bar{\lambda}_{1}}=1=e^{\lambda_{2}}$; thus $\lambda_{1}=2 k \pi i$ and $\lambda_{2}=2 j \pi i$ for some $k, j \in \mathbb{Z}$. This shows that $\lambda_{1} \equiv \lambda_{2}(\bmod 2 \pi i)$. Since $\sigma(a)-\sigma(b)$ is $2 \pi i$-congruence-free, we get $\lambda_{1}=\lambda_{2}$. Hence there is $k \in \mathbb{Z}$ such that $\sigma(c)=\{2 k \pi i\}$. Since $2 k \pi i$ is a simple zero of $g$, there is an entire function $h$ with

$$
g(\lambda)=h(\lambda)(\lambda-2 k \pi i)
$$

and $h(2 k \pi i) \neq 0$. This gives

$$
0=g(c)=h(c)(c-(2 k \pi i) \mathbf{1}) .
$$

Since $h(c)$ is invertible in $\mathcal{A}, c=(2 k \pi i) \mathbf{1}$.

As an immediate consequence of Theorem [6] we have the following well-known result:

Corollary 2. If $A$ and $B$ are bounded, self-adjoint operators on a complex Hilbert space and if $e^{A}=e^{B}$, then $A=B$.

Proof. From $\sigma(A), \sigma(A)-\sigma(B) \subseteq \mathbb{R}$ we see that $\sigma(A)$ and $\sigma(A)-\sigma(B)$ are $2 \pi i$ congruence-free. Theorem $[6$ now shows that $A-B=(2 k \pi i) I$ for some $k \in \mathbb{Z}$. From $A-B=(A-B)^{*}=-(2 k \pi i) I=-(A-B)$ we get $A=B$.

\section{REFERENCES}

[1] H. Heuser: Funktionalanalysis. 3rd ed., Teubner (1991). MR 94d:46001

[2] T. W. Palmer: Banach algebras and the general theory of *-algebras. Vol. I, Cambridge (1994). MR 95c:46002

[3] W. Rudin: Functional Analysis. McGraw-Hill (1973). [MR 51:1315

[4] Ch. Schmoeger: Remarks on commuting exponentials in Banach algebras. Proc. Amer. Math. Soc. 127 (1999), 1337-1338. MR 99h:46090

[5] E. M. E. Wermuth: Two remarks on matrix exponentials. Linear Algebra Appl. 117 (1989), 128-132. MR 90e:15019

[6] E. M. E. Wermuth: A remark on commuting operator exponentials. Proc. Amer. Math. Soc. 125 (1997), 1685-1688. MR 97g:39011

Mathematisches Institut I, Universität Karlsruhe, D-76128 Karlsruhe, Germany

E-mail address: christoph.schmoeger@math.uni-karlsruhe.de 